\title{
Niños (as) Económicamente Aventajados en el Espacio del Centro Comercial
}

\section{Upper and Upper Middle Class Children's Behaviour in Malls}

\author{
Salomón Magendzo* \\ Miguel Bahamondes*
}

\begin{abstract}
Resumen
La investigación se basó en el supuesto que la transmisión cultural y social de los adultos y los centros comerciales repercutiría en los comportamientos y los rituales que los niños(as) (que en el caso del presente estudio pertenecían a la clase alta y media alta), desplegarían en los espacios de consumo, en especial cuando éste es el centro comercial. Sin embargo, y en contradicción con lo anterior, también se postuló que, dada la etapa del desarrollo en que estos niños (as) se encuentran (9 a 11 años), se re-apropiarían del espacio en los términos de Certeaux (1996).

La metodología fue de carácter descriptivo, exploratorio y cuantitativa, utilizando la técnica de la observación no participante.

Los resultados dieron cuenta de diversos comportamientos de los niños (as) dentro del centro comercial, cuyo análisis permitió sustentar lo postulado respecto a la transmisión cultural de los adultos y del espacio comercial, pero no así la hipótesis de la re-apropiación.
\end{abstract}

Palabras Claves: Aprendizaje económico infantil, Transmisión cultural, Espacios urbanos

\section{Summary}

The following research was based on the assumption that the cultural and social transmission of adults and Malls, would rebound on the behaviors and rituals wich children (which in the case of the present study belonged to the upper and middleupper class), would displaye in consuming spaces, specifically in Mall. However, and contrary to the above, it was also claimed that given the stage of development in which these children are (9 to 11 years), they would re-appropriate the space as stated by Certeux (1996).

The methodology was descriptive, exploratory and quantitative, using the technique of non-participatory observation.

* Investigadores Universidad Academia de Humanismo Cristiano (UAHC), Santiago Chile, email:smagendzo@academia.cl,mbahamondes@academia.cl. Esta investigación fue desarrollada gracias al financiamiento otorgado por la UAHC a través de los Núcleos Temáticos de Investigación. 
The results accounted for diverse behaviors of the children inside the Mall, the analysis of which allowed to sustain what was postulated regarding the cultural transmission of the adults and the Mall, but not the hypothesis of the re-appropriation.

Key words: Economical child learning, Cultural transmission, Urban spaces.

\section{Introducción}

La presente investigación forma parte de una preocupación de los autores por la Psicología Económica centrada en los niños(as) de nueve a once años y definida ésta como la etapa del pensamiento económico en transición entre uno primitivo y otro de concepciones concretas dentro de los procesos de socialización económica y el consumo. En esta etapa los niños(as) hacen alusión a fuentes míticas o fantásticas para explicar el fenómeno en cuestión, pero también a ciertos conceptos aún poco elaborados (Denegri, 1997).

En un primer estudio Magendzo y Bahamondes ${ }^{1}$ hicieron alusión a Webley y Lea (1993) quienes postulaban que la Psicología Económica requiere progresos empíricos y teóricos en dos orientaciones: una apuntaría a la relación que existe entre la estructura económica de una sociedad con las ideas y el comportamiento económico de sus miembros más jóvenes, y la otra, al mundo económico que los mismos niños se construyen. El presente trabajo, va en esta dirección.

Interesa entregar los resultados y análisis del comportamiento de niños(as) de clase alta y media alta en un centro comercial emplazado en un barrio del sector pudiente de la ciudad de Santiago de Chile, rodeado de casas y edificios para este estrato social. El centro comercial se encuentra dentro de la propia ciudad y por tanto se puede acceder a pie o en auto.

Los hallazgos de las investigaciones respecto a la socialización del consumo apun- tan a una relación entre diferencias de nivel socio económico y la adquisición de habilidades y conductas como consumidor (Denegri et al, 1998). Éstas aparecen más tempranas entre los jóvenes del estrato social más alto, particularmente entre los varones. Al provenir de familias de ingresos considerablemente mejores tienen un rango más grande de experiencias económicas. Los mismos hallazgos encontrados entre los jóvenes, es posible hacerlos extensivos a niños(as) pre púberes de familias adineradas, como son los niños(as) de este estudio.

En la presente investigación se postulaba, primero, que la transmisión cultural y social de los adultos sobre los niños (as) se verá reflejada en los comportamientos y rituales que estos despliegan en los espacios de consumo, en especial cuando éste es un centro comercial. Por tanto, observar sus conductas en estos ambientes debiera permitir deducir las maneras como estos niños(as) han sido socializados por los adultos cuando pertenecen a los estratos altos de la sociedad.

También el centro comercial (con sus propias características, típicas de un espacio simbólico de la modernidad y descrito en esos términos, y en forma tan gráfica, por diferentes autores, (Sarlo1996, Moulián 1997)), transmitiría a los niños(as) comportamientos específicos respecto al espacio. Entonces lo que hacen los niños (as) en los centros comerciales es producto de la socialización económica que estos centros ejercen sobre los niños (as) y aquello que los adultos han inculcado en éstos.

1 En el trabajo citado (La participación de los niños pobres en el 'espectáculo' del consumo) los autores se abocan al registro y análisis del comportamiento observable de niños(as) pobres en un centro comercial del centro de la cuidad donde acuden personas de clase media y de escasos recursos. Los resultados in extenso fueron publicados por Magendzo y Bahamondes en la revista Castalia número 3, volumen 1, año 2002, de la Escuela de Psicología de la Universidad Academia de Humanismo Cristiano. 
Sin embargo, además, y quizás lo fundamental, postulamos, a modo de hipótesis, que dada la etapa de desarrollo en que se encuentran los niños (as) de alguna manera ellos se reapropiarían, en el lenguaje de Certeau, del espacio del Mall. Cuando se dice reapropiación se apunta a una conducta que opera como un rechazo a las influencias sociales tanto de la casa como del centro comercial y en su lugar se observará una construcción original de parte de los niños (as) del espacio.

Teniendo en cuenta lo anterior es obvio pensar que los niños (as) van adquiriendo sus comportamientos económicos a partir de la influencia social y de la posición que ocupan dentro de la sociedad. Por tanto, siguiendo lo expresado por Amar (2002), se plantea que el medio fundamental, a la hora de explicar como se representa la realidad económica en la mente infantil, es la influencia social y no sólo aquello provisto por el enriquecimiento de las estructuras cognitivas. Es decir, son las experiencias del niño (a) al estar en contacto con el contexto social en interacción con el desarrollo cognitivo, lo que permite comprender la formación de las ideas, nociones y comportamientos económicos de la infancia.

Lo expuesto queda teóricamente definido con Delval (1989) cuando éste determina como los niños (as) llegan a representarse la realidad social y elabora tres conceptos que permiten el análisis y la comprensión de los comportamientos sociales de los niños (as): A, son las normas y valores, los que prescriben lo deseable y lo que no lo es, desde el punto de vista social, constituyéndose en reguladores de la conducta. B, son las informaciones diversas que las personas deben adquirir para ubicarse en el mundo que les toca vivir. A y B son adquiridas por los niños (as), primero a través de la imitación o la enseñanza explícita entregada por los adultos, los medios de comunicación, la escuela o cualquier otro símbolo creado por el medio, como ser los centros comerciales. O segundo, los niños (as) la buscan por sí mismo en su contacto con la realidad. C, son las explicaciones acerca del por qué de las cosas y del funcionamiento social, los que están mayormente ausentes de la transmisión social y los cuales el niño(a) debe construir con el bagaje intelectual que dispone en cada una de las etapas del desarrollo en que se encuentra conforme a su edad. Estas explicaciones muchas veces divergen de las de los adultos. Conocer el A y el B que manejan los niños(as) nos permite conocer las características sociales del grupo de pertenencia. Entender C, nos facilita saber de las formas en que ellos construyen el conocimiento y las tendencias evolutivas presentes en el proceso. Como lo afirma Amar (2002) el niño (a) crea ideas de cómo funcionan las cosas de su entorno, y al confrontar éstas con la realidad puede llegar a darse cuenta de que no son así. El conflicto que eventualmente se crea en esa contradicción le obliga a resolverlo de una u otra manera. Más aún podemos agregar que a medida que el entorno, y en el caso de nuestra investigación el centro comercial, le es más propio al niño (a), se siente perteneciendo a él y con menos contradicciones, éste absorberá con mayor facilidad mensajes tácitos que el medio le provee.

Delval (1989) sustenta que los pilares organizativos del esquema global de las representaciones del mundo social son el orden político y el orden económico. Si sobre estos dos órdenes giran los principales problemas que conciernen a la organización de la sociedad, no podemos menos que afirmar la trascendencia que tiene acumular conocimientos en esta perspectiva.

Los investigadores cuando se interesan por saber cómo entiende y adquiere el niño (a) los conceptos económicos como ser el dinero, la banca, la movilidad social, el trabajo, el desempleo, etc., se preocupan por determinar el entendimiento que los niños (as) tienen, a diferentes edades, acerca de estos conceptos. La presente investigación por el contrario, más bien centra su atención en los aspectos no verbales, conductuales, de acción, que desplie- 
gan los niños (as) en los espacios económicos y de ahí lograr inferir conclusiones y predicciones del futuro social de estos.

Se postula que la observación de los aspectos psicomotores que los niños (as) exhiben espontáneamente, permite superar, parcialmente, la dificultad que se les presenta a los investigadores cuando intentan conocer los conceptos económicos de los niños (as) a través de la comunicación verbal. Estos conceptos están arraigados más bien en forma tácita, no verbal. Son éstos los que en última instancia permiten tomar las decisiones y desplegar los comportamientos de una manera especifica en el mundo social. Es decir, se postula que es más bien lo no consciente, lo adquirido sin darnos mayor cuenta al estar en contacto permanente con el entorno y la realidad social llena de significados-, la que nos impregna tácitamente y la que prevalece en nosotros, superando las conceptualizaciones verbales (políticas y económicas) adquiridas a través del tiempo. En este sentido cabe mencionar el estudio de Bonn y Webley (2000) citado por Amar (2002), quienes encontraron que los niños chinos de Hong Kong superaban a los niños escoceses y japoneses en la comprensión del dinero y su función, así como del funcionamiento bancario; y que ésta mayor comprensión económica se debe en gran medida al alto nivel de socialización económica a la que se ven expuestos desde temprana edad por el ethos comercial tan amplio que existe en su sociedad.

En la misma línea de lo anterior Bourdieu sostiene lo siguiente: "Las disposiciones económicas más fundamentales, necesidades, preferencias, propensiones, no son exógenas, esto es, dependientes de una naturaleza humana universal, sino endógenas y dependientes de una historia, que es la misma del cosmos económico en que se exigen y recompensan. Vale decir que, contra la distinción canónica entre los fines y los medios, el campo impone a todos, pero en grados diversos según su posición y sus capacidades económicas, no sólo los medios 'razonables', sino los fines a saber, el enriquecimiento individual de la acción económica" (cursiva del autor)" (2002)

Y continúa el mismo autor:

"La economía de las prácticas económicas, esa razón inmanente a las prácticas, no tiene su principio en 'decisiones' de la voluntad y la conciencia racionales o en determinaciones mecánicas originadas en poderes exteriores, sino en las disposiciones adquiridas por medio de los aprendizajes asociados a una prolongada confrontación con las regularidades del campo; esas disposiciones son capaces de generar, incluso al margen de cualquier cálculo consciente, conductas y hasta previsiones que más vale llamar razonables que racionales, aun cuando su conformidad con las estimaciones del cálculo nos incline a pensarlas y tratarlas como producto de la razón calculadora." (Cursiva del autor) (Ibid)

\section{Objetivos}

Los objetivos del estudio estuvieron centrados en:

1. Describir antecedentes generales de la visita de los niños(as) al centro comercial.

2. Describir las relaciones entre niños(as) y sus acompañantes en el centro comercial.

3. Describir las conductas de los niños(as) al llegar a distintos espacios del centro comercial.

4. Describir la conducta de los niños(as) en el espacio de compras.

5. Describir el acto de compra.

6. Describir en particular la conducta de los niños(as) en espacios de alimento y entretenimiento.

7. Determinar la asociación estadística entre las variables relativas a espacios de recorrido y consumo en el centro comercial, y las características y conductas de niños(as) y acompañantes. 


\section{Metodología}

\section{Método}

El estudio fue de carácter eminentemente descriptivo, exploratorio y de orden cuantitativo. Interesó registrar, a través de la observación no participante (técnica no intrusiva), el comportamiento de los niños (as), en un espacio de consumo (centro comercial), sin que mediara una relación de dialogo entre los observadores y estos.

\section{Procedimiento}

\subsection{INSTRUMENTO}

Por tratarse de un registro etnográfico del abanico de conductas desplegadas por los niños en un espacio de consumo, no se empleó una pauta de observación estructurada. El observador tenía como única instrucción el registrar el conjunto de comportamientos que mostraba el niño respecto al espacio, a su o sus acompañantes, y a los objetos.

\subsection{OBSERVACIÓN}

Los observadores ${ }^{3}$ efectuaron un registro etnográfico del comportamiento mostrado por los niños (as) y de los que lo acompañaban, si era el caso, en un área o espacio del centro comercial, delimitado de antemano por los investigadores. Se distinguieron cuatro espacios de observación: entretención, comida, vestuario y varios. ${ }^{4}$ El centro comercial seleccionado para llevar adelante la observación, está ubicado en el sector oriente de la ciudad de Santiago donde concurre gran cantidad de publico de clase media alta y alta. El centro comercial se encuentra relativamente integrado a las calles adyacentes.

El registro se iniciaba una vez que los niños (as) "seleccionados" entraban en el espacio de observación y se mantenía durante todo el tiempo que estaban en él. La observación concluía en el momento que el niño(a) salía del área delimitada.

Las personas a cargo del registro fueron cuatro. En reuniones con los investigadores se discutió y concordó los criterios que se emplearían para estimar cuales de los niños(as) eran de clase media alta o alta y por tanto sujetos del estudio. Finalmente, se identificaron los lugares y la posición que ocuparían los observadores en el espacio.

La observación se prolongó por cuatro meses (diciembre del 2002, enero, marzo y abril 2003), y para ello se empleó la siguiente rutina:

a) Las observaciones se realizaron durante dos momentos: a mediado y a fin de cada mes. b) En ambos momentos se concurrió al lugar de observación un día a mitad de semana (miércoles) y el otro a fin de ésta (sábado). c) Con el propósito de registrar el flujo normal de niños (as) que transitaban por el mall, en cada uno de los días se efectuó la observación en tres diferentes oportunidades: por la mañana, a mediodía y por la tarde, durante dos horas cada vez. d) Para recabar la información se estableció una pauta donde se anotó el día de la observación, hora de inicio y de término de ésta y el lugar donde se había realizado. A continuación el observador registraba los comportamientos $\mathrm{u}$ otros da-

\footnotetext{
2 La metodología de la presente investigación fue similar a la utilizada en el estudio con niño(a)s(as) pobres, anteriormente mencionado. La razón de ello radica en que en una fase posterior se hará un estudio comparativo entre los comportamientos de los niño(a)s(as) pobres y los aventajados económicamente, que dará origen, a su vez, a una tercera publicación.

3 Las cuatro personas que estuvieron a cargo del registro en terreno tenían experiencia previa en el trabajo con niño(a)s(as) lo que resultaba fundamental para el estudio, puesto que la "selección" de los niño(a)s que debían ser observados sólo se basó en la presencia de rasgos que "caracterizan" la condición de niño(a) pobre.

4 Los lugares son de características similares a los del primer estudio.
} 
tos obtenidos, absteniéndose de incluir interpretaciones. El registro debía mantener la secuencia de lo observado desde el momento que el niño(a) y sus acompañantes (si era el caso) entraban al espacio de observación específico hasta que salían de él, o no estaban a la vista del observador.

\section{Procesamiento de los datos y análisis.}

Para el procesamiento y análisis de la información se procedió a efectuar una lectura acuciosa de los registros y a continuación se elaboró una codificación de las características de los niños(as) y conductas desplegadas por éstos. Posteriormente, se procedió a vaciar la información de cada uno en una matriz que contenía los indicadores de la codificación elaborada. Con el fin de apoyar el trabajo de vaciado de la información en la matriz, se elaboró un glosario de criterios y definiciones de cada uno de los indicadores. Cabe destacar que, dada la metodología utilizada, la información obtenida variaba numéricamente para cada indicador. Finalmente con una muestra del 10\% de los registros seleccionados al azar, se ratificó a través de una codificación doble, la certeza que se tuvo en adjudicar las distintas observaciones en las correspondientes categorías de la codificación.
Para el análisis de los datos se utilizó el programa computacional SPSS.

\section{Resultados}

\section{Analisis Descriptivo}

\section{Antecedentes Generales}

\subsection{Período, Horario y Tipo de Espacio de Observación}

Doscientos setenta y ocho niños y niñas fueron observados. Los períodos de registro, en cuanto si se realizaron al comienzo del mes o en la mitad o a comienzo de la semana o en la mitad fueron proporcionalmente similares (alrededor del 25\%) El horario de observación en el que se presentó la mayor frecuencia de niños(as) fue en la tarde (52\%), distribuyéndose el resto en la mañana y medio día en porcentaje semejante $(25 \%)$. Respecto al porcentaje de niños y niñas que frecuentaron los cuatro espacios asignados por la investigación para la observación en el mall (comida, vestuario, recreación, varios) fueron similares en cada uno de ellos (alrededor del $25 \%$ ) (tabla 1)

Tabla 1. Período, horario y tipo de espacio de observación

\begin{tabular}{|l|c|c|c|c|c|c|}
\hline \multirow{2}{*}{ Sexo } & \multicolumn{4}{|c|}{ Lugares de observación } & \multicolumn{2}{c|}{ Total } \\
\cline { 2 - 7 } & Comida & Vestuario & Recreación & Varios & $N^{o}$ & $\%$ \\
\hline Hombres & 43 & 39 & 46 & 35 & 163 & 58.6 \\
\hline Mujeres & 32 & 34 & 14 & 35 & 115 & 41.4 \\
\hline Total & 75 & 73 & 60 & 70 & 278 & 100.0 \\
\hline$\%$ & 27.0 & 26.3 & 21.6 & 25.2 & & \\
\hline
\end{tabular}

\subsection{Características de Niños y Niñas}

Fueron observados ciento sesenta y tres niños (59\%) y ciento quince niñas (41\%) Los niños y niñas en un $70 \%$ vestían normalmente; sólo el $10 \%$ fue calificado en la categoría de vestimenta extravagante, y similar porcen- taje en la categoría de "marca". El fenotipo predominante fue trigueño (45\%), sin embargo también se observaron morenos (27\%) y rubios (25\%). Los niños y niñas tendieron a ser más bien delgados (44\%), un 11\% fue calificado como obesos. 


\section{RELACIÓN NiÑOS (AS) Y ACOMPAÑANTES}

\subsection{Características de los Acompañantes}

Los niños y niñas llegaron al lugar en alta proporción, acompañados, ya sea por una persona $(63 \%)$ o de más de una $(31 \%)$. Escasamente vinieron solos (6\%). Fueron acompañados, en un alto porcentaje más bien por mujeres $(41 \%) \mathrm{u}$ hombres y mujeres $(25,2 \%)$.
Los niños (as) vinieron exclusivamente con acompañantes hombres en un $29 \%$. El tipo de acompañantes fue principalmente adulto $(52 \%)$ o heterogéneos, es decir de todas la edades (25\%). Los niños(as) acompañantes (solos) de la misma edad o menores fueron escasos (7\%) al igual que los adultos mayores $(2 \%)$. Los acompañantes jóvenes fueron algo más (13\%) (tabla 2).

Tabla 2. Características de los acompañantes

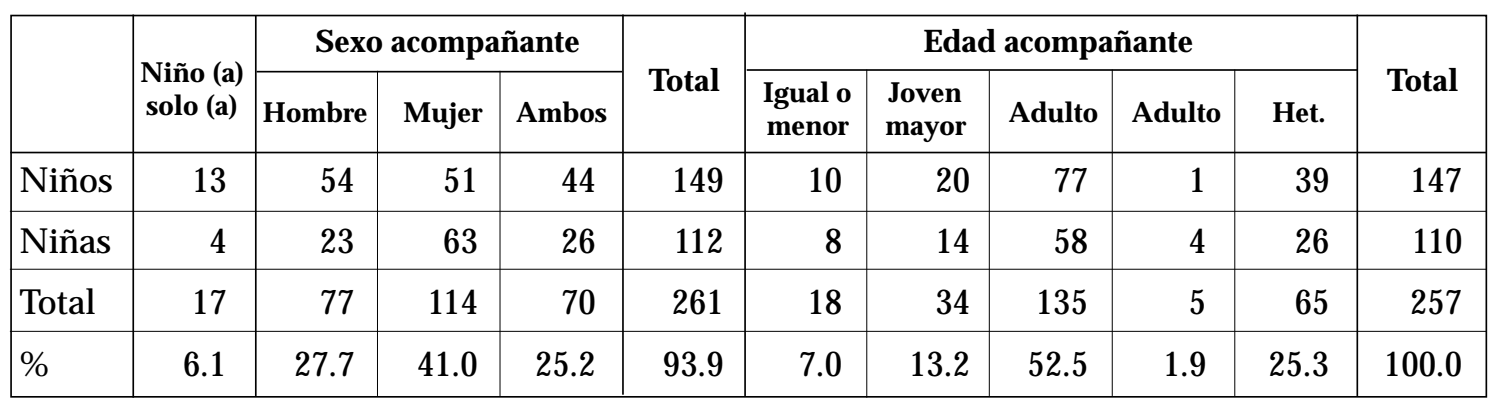

\subsection{Conducta de los (as) Niños (as)} Hacia los Acompañantes

Un $41 \%$ de los niños (as) tuvo una conducta pasiva frente al acompañante lo que se expresó en que éstos no manifestaron re- querimientos visibles, ni conversación con los acompañantes. Por otro lado, un $49 \%$ se acercó al espacio dialogando con quienes los traían. Un 5\% mostró evidentes gestos de buscar conmover al acompañante para que hiciera algo a su favor (tabla 3 ).

Tabla 3. Conducta de los (as) niños (as) hacia los acompañantes

\begin{tabular}{|l|c|c|c|c|c|c|}
\hline \multirow{2}{*}{$\begin{array}{c}\text { Tipo } \\
\text { conducta }\end{array}$} & \multicolumn{2}{|c|}{ Total } & \multicolumn{2}{c|}{ Hombres } & \multicolumn{2}{c|}{ Mujeres } \\
\cline { 2 - 7 } & $\mathbf{N}^{\mathbf{0}}$ & $\mathbf{\%}$ & $\mathbf{N}^{\mathbf{0}}$ & $\mathbf{\%}$ & $\mathbf{N}^{\mathbf{0}}$ & $\mathbf{\%}$ \\
\hline Pasiva & 99 & 41.1 & 66 & 46.5 & 33 & 33.3 \\
\hline Diálogo & 12 & 49.8 & 63 & 44.4 & 57 & 57.8 \\
\hline Súplica & 12 & 5.0 & 7 & 4.9 & 5 & 5.1 \\
\hline Compulsiva & 2 & 0.8 & 2 & 1.4 & 2 & 2.0 \\
\hline Manipuladora & 4 & 1.7 & 1 & 0.7 & 3 & 3.0 \\
\hline Múltiple & 4 & 1.7 & 3 & 2.1 & 1 & 1.0 \\
\hline Total & 241 & 100.0 & 142 & 100.0 & 99 & 100.0 \\
\hline
\end{tabular}

Además, los niños (as) frente a los requerimientos de los acompañantes en la gran mayoría (83\%) acataban sin dar muestra de desagrado manifiesto o algún gesto que denotara contrariedad o intento de reiterar la conducta. Solo un 5\% mostró enfado o eno- 
jo, es decir, molestia ante la conducta del acompañante; y un $8 \%$ indiferencia, expresada en que el niño(a) no tomaba en cuenta la reacción del acompañante o no manifestaba mayor preocupación por éste o ésta.

\section{Conducta de los Niños (AS) al Llegar A Los Espacios}

\subsection{Niño (a) Solo (a)}

En los niños(as) que transitaban solos, se observó en un $47 \%$ una conducta indiferen- te-pasiva frente al espacio; o sea, circulaban por el lugar pero no prestaban mayor atención al espacio por el cual transitaban. Otro $50 \%$ mostró un comportamiento activo, de acercamiento y entusiasmo.

\section{2. Niño (a) Acompañado}

En los niños(as) que transitaban acompañados se observó, en un $33 \%$, una conducta indiferente-pasiva frente al espacio. Otro $66 \%$ mostró un comportamiento activo (tabla 4).

Tabla 4. Conducta de los niños (as) al llegar a los espacios

\begin{tabular}{|l|r|r|r|r|r|r|r|r|}
\hline \multirow{2}{*}{$\begin{array}{c}\text { Tipo } \\
\text { comportamiento }\end{array}$} & \multicolumn{4}{|c|}{ Niños (as) solos (as) } & \multicolumn{4}{c|}{ Niños (as) acompañados (as) } \\
\cline { 2 - 10 } & Niños & Niñas & Total & \multicolumn{1}{c|}{$\%$} & Niños & Niñas & Total & \% \\
\hline Indiferente & 4 & 3 & 7 & 41.2 & 21 & 9 & 30 & 12.1 \\
\hline Activa & 8 & 1 & 9 & 52.9 & 89 & 74 & 165 & 66.0 \\
\hline Pasiva & 1 & - & 1 & 5.9 & 33 & 19 & 52 & 21.1 \\
\hline Evitativa & - & - & - & - & 2 & - & 2 & 0.8 \\
\hline Total & 13 & 4 & 17 & 100.0 & 145 & 102 & 247 & 100.0 \\
\hline
\end{tabular}

\section{Conducta del Niño (a) en el Espacio DE COMPRA}

Diversas fueron las conductas de los niños (as) en el espacio de compra. El $41 \%$ tuvo una conducta "predefinida-directa", es decir, ingresaban al espacio y se dirigían al lugar de su interés en forma inmediata. Otro $32 \%$ lo hacia "predefinido-con recorrido", lo que significo que se dirigían al lugar de su interés pero en el trayecto miraban hacia otros espacios o sectores. Finalmente, un 20\% lo hacia como un "reconocimiento simple", lo que equivale a realizar un recorrido sin un norte claro y durante el cual se van observando distintos objetos; $y$ un $7 \%$ con un "reconocimiento-reiterativo" donde los niños (as) iniciaban un recorrido que los llevaba a detenerse por un tiempo variable frente a objetos que llamaban su atención pudiendo volver sobre ellos (tabla 5).

Tabla 5. Conducta del niño (a) en el espacio de compra

\begin{tabular}{|l|r|r|r|r|r|r|}
\hline \multirow{2}{*}{ Tipo de comportamiento } & \multicolumn{2}{|c|}{ Total } & \multicolumn{2}{c|}{ Hombres } & \multicolumn{2}{c|}{ Mujeres } \\
\cline { 2 - 7 } & $\mathbf{N}^{\mathbf{0}}$ & $\mathbf{\%}$ & $\mathbf{N}^{\mathbf{0}}$ & $\mathbf{\%}$ & $\mathbf{N}^{\mathbf{0}}$ & $\mathbf{\%}$ \\
\hline Predefinido-directo & 97 & 70.3 & 51 & 73.9 & 46 & 66.7 \\
\hline Predefinido-con recorrido & 8 & 5.8 & 4 & 5.8 & 4 & 5.8 \\
\hline Recorrido-simple & 30 & 21.7 & 12 & 17.4 & 18 & 26.1 \\
\hline Recorrido-reiterado & 3 & 2.2 & 2 & 2.9 & 1 & 1.5 \\
\hline Total & 138 & 100.0 & 69 & 100.0 & 69 & 100.0 \\
\hline
\end{tabular}


El 67\% tuvo una conducta activa y autónoma hacia los objetos, es decir, se interesó por ellos sin mayor inducción y como producto de una acción individual. Sólo el 26\% fue pasivo, expresado esto en un comportamiento de poco entusiasmo frente a los objetos, y un 6\% necesitó de apoyo e inducción para interesarse en ellos.
Una vez frente a los objetos, el 78\% de los niños(as) los tomó, manipuló y examinó su funcionamiento. Un $21 \%$ se quedó lejano o pasivo, es decir tomaban el objeto pero no lo manipulaban, por el contrario, mas bien tenían un tratamiento cuidadoso frente a ellos.

Tabla 6. Conducta del niño (a) frente a los objetos y tratamiento de los objetos

\begin{tabular}{|l|l|r|r|r|r|r|r|}
\hline \multirow{4}{*}{ Variable } & Tipo de & \multicolumn{2}{|c|}{ Total } & \multicolumn{2}{c|}{ Hombres } & \multicolumn{2}{c|}{ Mujeres } \\
\cline { 3 - 8 } & conducta & $\mathbf{N}^{\mathbf{0}}$ & $\mathbf{\%}$ & $\mathbf{N}^{\mathbf{0}}$ & $\mathbf{\%}$ & $\mathbf{N}^{\mathbf{0}}$ & $\mathbf{\%}$ \\
\hline \multirow{3}{*}{$\begin{array}{l}\text { Conducta } \\
\text { frente a } \\
\text { objeto }\end{array}$} & Pasiva & 127 & 87.0 & 77 & 83.7 & 50 & 92.6 \\
\cline { 2 - 8 } & Autónoma & 8 & 5.5 & 5 & 5.4 & 3 & 5.6 \\
\cline { 2 - 8 } & Inducida & 11 & 7.5 & 10 & 10.9 & 1 & 1.8 \\
\cline { 2 - 8 } & Total & 146 & 100.0 & 92 & 100.0 & 54 & 100.0 \\
\hline \multirow{4}{*}{$\begin{array}{l}\text { Tratamiento } \\
\text { del objeto }\end{array}$} & Lejano & 80 & 44.4 & 52 & 47.7 & 28 & 39.4 \\
\cline { 2 - 8 } & Activo & 62 & 34.4 & 35 & 32.1 & 27 & 38.0 \\
\cline { 2 - 8 } & Pasivo & 38 & 21.1 & 22 & 20.2 & 16 & 22.6 \\
\cline { 2 - 8 } & Total & 180 & 100.0 & 109 & 100.0 & 71 & 100.0 \\
\hline
\end{tabular}

El 48\% mientras estaban frente a los objetos de su interés, conversaban relajadamente con sus acompañantes, inquiriendo todo el tiempo características acerca de los objetos. Otro $17 \%$, a pesar que no dialogaba con el acompañante, manifestaron una actitud de contacto adecuado. Por otro lado, en un $28 \%$ de los registros, entre los niños(as) y acompañantes, no hubo ninguna interacción, predominando la indiferencia. Además en estos casos, él (la) o los(as) acompañantes, no dio muestra de mayor atención o vigilancia sobre los niños(as).

\section{ACTO DE COMPRA}

Un 58\% de los niños(as) no hizo ninguna compra; y entre los que compraron (42\%) un $19 \%$ adquirió cosas de buena calidad o en cantidad considerable y un $23 \%$ pocos objetos o de escaso valor. En alrededor de un $50 \%$ no participaron en forma activa en el pago del bien, es decir no fueron considerados en el cierre de la transacción; el otro 50\% sí estuvo incluido, ya sea en forma real o simulada.

$\mathrm{Al}$ retirarse del lugar de compra un $71 \%$ se mostró indiferente, es decir no dieron muestra evidente de agrado o desagrado. Sólo un 20\% abandonó el lugar dando muestras claras de alegría o satisfacción, como riéndose o saltando. En un 7\% se observó tristeza y frustración, pero sin acompañar ésta con conductas de rebeldía o desagrado (tabla 7). 
Tabla 7. Comportamiento de los niños(as) respecto a la compra, participación en el pago y al retirarse del lugar

\begin{tabular}{|c|c|c|c|c|c|c|c|}
\hline \multirow[t]{2}{*}{ Variable } & \multirow{2}{*}{\begin{tabular}{c|} 
Tipo \\
conducta
\end{tabular}} & \multicolumn{2}{|c|}{ Total } & \multicolumn{2}{|c|}{ Hombres } & \multicolumn{2}{|c|}{ Mujeres } \\
\hline & & $\mathrm{N}^{\mathrm{o}}$ & $\%$ & $\mathrm{~N}^{\mathrm{o}}$ & $\%$ & $\mathrm{~N}^{\mathrm{o}}$ & $\%$ \\
\hline \multirow[t]{4}{*}{ Monto compra } & Máxima & 40 & 18.7 & 25 & 19.2 & 15 & 17.9 \\
\hline & Mínima & 50 & 23.4 & 31 & 23.8 & 19 & 22.6 \\
\hline & Ninguna & 124 & 57.9 & 74 & 56.9 & 50 & 59.5 \\
\hline & Total & 214 & 100.0 & 130 & 100.0 & 84 & 100.0 \\
\hline \multirow[t]{3}{*}{ Participación pago } & Inclusión & 48 & 46.6 & 38 & 57.6 & 10 & 27.0 \\
\hline & Exclusión & 55 & 53.4 & 28 & 42.4 & 27 & 73.0 \\
\hline & Total & 103 & 100.0 & 66 & 100.0 & 37 & 100.0 \\
\hline \multirow{5}{*}{$\begin{array}{l}\text { Comportamiento al } \\
\text { retirarse del lugar }\end{array}$} & Alegre & 42 & 19.4 & 22 & 16.9 & 20 & 22.9 \\
\hline & Triste & 15 & 6.9 & 9 & 6.9 & 6 & 6.9 \\
\hline & Molesto & 5 & 2.3 & 5 & 3.8 & & \\
\hline & Indiferente & 155 & 71.4 & 94 & 72.3 & 61 & 70.2 \\
\hline & Total & 217 & 100.0 & 130 & 100.0 & 87 & 100.0 \\
\hline
\end{tabular}

\section{Conductas Específicas Frente aL Consumo de Alimentos}

En un mayor porcentaje son los niños(as) los que indujeron a los acompañantes hacia el espacio de los alimentos del mall, ya sea manifestando verbal o gestualmente su interés o sin estas expresiones tan directas (57\%); el porcentaje restante $(43 \%)$ muestra la conducta inversa: son los acompañantes los que llevan a los niños(as) a este espacio.

El acto de consumo de alimentos se realizó en un $86 \%$ como algo colectivo, es decir, el niño(a) se acercó y participó del consumo como integrante de un grupo que en forma conjunta se involucró en la acción. Un 14\% circuló en este espacio o solo, o se separó de sus acompañantes, y se dirigió al lugar de alimentación en forma autónoma. El $66 \%$ participó de manera activa en la discusión para elegir qué comer. Un $14 \%$ no participó en la elección y aceptó lo que los acompañantes seleccionaron, empero estuvieron atentos al proceso. Un 19\% se desentendieron de la elección.
En cuanto a la forma en que se llevó a cabo el acto de consumo, un porcentaje significativo de los comensales (41\%) intercambiaban opiniones, es decir conversaban amenamente. Otro $22 \%$ acompañó el intercambio de opiniones con formas variadas de entretención, jugando y haciendo bromas. Hubo un $18 \%$ que se dedicó a comer en silencio, y en un $15 \%$ los comensales no establecieron diálogos entre ellos, solo interés por cosas del entorno y no por las personas. En un bajo $4 \%$ el consumo ocurrió en un ambiente de órdenes y autoritarismo de parte de los adultos, donde se evidenció tensión y conflictos. Finalmente, el tiempo destinado a comer se distribuyó relativamente parejo en tres categorías: breve-rápido (38\%), prolongado $(29 \%)$ e intermedio $(33 \%)$; y en un alto porcentaje $(80 \%)$ el grupo de comensales terminado el consumo retiraron las bandejas de la mesa, depositaron los restos en el contenedor de basura y colocaron las bandejas en el lugar dispuesto para ello (tabla 8). 
Tabla 8. Conductas específicas frente al consumo de alimentos

\begin{tabular}{|c|c|c|c|c|c|c|c|}
\hline \multirow[t]{2}{*}{ Variable } & \multirow[t]{2}{*}{ Tipo conducta } & \multicolumn{2}{|c|}{ Total } & \multicolumn{2}{|c|}{ Hombres } & \multicolumn{2}{|c|}{ Mujeres } \\
\hline & & $\mathbf{N}^{\mathbf{o}}$ & $\%$ & $\mathbf{N}^{\mathbf{o}}$ & $\%$ & $\mathbf{N}^{\circ}$ & $\%$ \\
\hline \multirow[t]{4}{*}{$\begin{array}{c}\text { Disposición } \\
\text { consumo alimentos }\end{array}$} & $\begin{array}{l}\text { Inducido } \\
\text { por adulto }\end{array}$ & 21 & 42.9 & 14 & 48.3 & 7 & 35.0 \\
\hline & $\begin{array}{l}\text { Inducido por } \\
\text { niño (directo) }\end{array}$ & 16 & 32.7 & 8 & 27.6 & 8 & 40.0 \\
\hline & $\begin{array}{c}\text { Inducido por } \\
\text { niño (secundario) }\end{array}$ & 12 & 24.5 & 7 & 24.1 & 5 & 25.0 \\
\hline & Total & 49 & 100.0 & 29 & 100.0 & 20 & 100.0 \\
\hline \multirow[t]{4}{*}{ Elección alimento } & Activa & 41 & 66.1 & 24 & 68.6 & 17 & 63.0 \\
\hline & Pasiva & 9 & 14.5 & 4 & 11.4 & 5 & 18.5 \\
\hline & Neutra & 12 & 19.4 & 7 & 20.0 & 5 & 18.5 \\
\hline & Total & 62 & 100.0 & 35 & 100.0 & 27 & 100.0 \\
\hline \multirow[t]{6}{*}{ Forma consumo } & Dialogada & 22 & 40.7 & 15 & 45.5 & 7 & 33.4 \\
\hline & Lúdica & 12 & 22.2 & 6 & 18.2 & 6 & 28.6 \\
\hline & Silenciosa & 10 & 18.5 & 6 & 18.2 & 4 & 19.0 \\
\hline & Conflictiva & 2 & 3.7 & 2 & 6.1 & & \\
\hline & Indiferencia & 8 & 14.8 & 4 & 12.1 & 4 & 19.0 \\
\hline & Total & 54 & 100.0 & 33 & 100.0 & 21 & 100.0 \\
\hline \multirow[t]{4}{*}{ Fin del consumo } & Retira bandeja & 33 & 80.4 & 23 & 82.1 & 10 & 76.9 \\
\hline & No retira bandeja & 4 & 9.8 & 3 & 10.7 & 1 & 7.7 \\
\hline & \begin{tabular}{|c}
$\begin{array}{c}\text { No retira bandeja- } \\
\text { mesa sucia }\end{array}$ \\
\end{tabular} & 4 & 9.8 & 2 & 7.2 & 2 & 15.4 \\
\hline & Total & 41 & 100.0 & 28 & 100.0 & 13 & 100.0 \\
\hline
\end{tabular}

\section{Conducta Especificas Frente a} Espacios de Juegos (Máquinas de Juegos)

Respecto a como los niños(as) adquirían la fichas para jugar en las máquinas se observó que el $49 \%$ se lo solicitaba al acompañante y el $39 \%$ las obtuvo con dinero que ellos traían. El 4\% tenían tarjetas para jugar.

En cuanto a la manera de jugar, el $78 \%$ lo hacia en forma real, es decir, efectivamente podía operar la máquina al activarla introduciendo fichas. Solo un $2 \%$ lo hizo de manera simulada, lo que se expresaba en que el niño(a) interactuaba con la máquina sin acti- varla. Hubo también un $20 \%$ que se limitó a observar como otros jugaban.

En la interacción con las máquinas las conductas se agruparon en dos: tranquila, $52 \%$, es decir sin mostrar mayores gestos de agrado o entusiasmo, y efusiva, $48 \%$, donde los niños(as) sí se manifestaron contentos.

Finalmente, un alto porcentaje (78\%) no se preocupó por impresionar a la gente que los rodeaba, mas bien se mostraron indiferentes. Solo un $12 \%$ mostró deseos de impresionar a otros con sus habilidades frente a las máquinas (tabla 9). 
Tabla 9. Comportamiento de los niños(as) en el espacio de recreación (juegos)

\begin{tabular}{|c|c|c|c|c|c|c|c|}
\hline \multirow[t]{2}{*}{ Variable } & \multirow[t]{2}{*}{ Tipo conducta } & \multicolumn{2}{|c|}{ Total } & \multicolumn{2}{|c|}{ Hombres } & \multicolumn{2}{|c|}{ Mujeres } \\
\hline & & $\mathbf{N}^{\mathbf{o}}$ & $\%$ & $\mathrm{~N}^{\mathrm{o}}$ & $\%$ & $\mathbf{N}^{o}$ & $\%$ \\
\hline \multirow[t]{5}{*}{ Acceso a juegos } & $\begin{array}{l}\text { Solicita dinero } \\
\text { acompañante }\end{array}$ & 25 & 49.0 & 16 & 42.1 & 9 & 69.2 \\
\hline & Dinero propio & 20 & 39.2 & 19 & 50.0 & 1 & 7.7 \\
\hline & Regalada & 4 & 7.8 & 1 & 2.6 & 3 & 23.1 \\
\hline & Trae ficha & 2 & 3.9 & 2 & 5.3 & & \\
\hline & Total & 51 & 100.0 & 38 & 100.0 & 13 & 100.0 \\
\hline \multirow{4}{*}{$\begin{array}{l}\text { Interacción } \\
\text { con máquina }\end{array}$} & Pasiva & 12 & 20.0 & 10 & 21.7 & 2 & 14.3 \\
\hline & Simulada & 1 & 1.6 & 1 & 2.2 & & \\
\hline & Real & 47 & 78.3 & 35 & 76.1 & 12 & 85.7 \\
\hline & Total & 60 & 100.0 & 46 & 100.0 & 14 & 100.0 \\
\hline \multirow[t]{4}{*}{ Actitud ante público } & Arrogancia & 6 & 12.3 & 3 & 8.1 & 3 & 25.0 \\
\hline & Indiferencia & 36 & 73.5 & 29 & 78.4 & 9 & 75.0 \\
\hline & Humildad & 5 & 10.2 & 5 & 13.5 & & \\
\hline & Total & 49 & 100.0 & 37 & 100.0 & 12 & 100.0 \\
\hline
\end{tabular}

\section{Otros Resultados}

La investigación se orientó, además, a determinar la asociación estadística entre algunas de las variables descritas más arriba.

Se encontró dependencia estadística entre las variable que se indican a continuación.

1. Espacios predeterminados del estudio (comida, vestuario, recreación y varios) y el sexo. Significativamente se observó más varones que niñas frecuentando el espacio de recreación (28\% y $12 \%$ respectivamente). En los restantes espacios la relación es justamente la opuesta (comida $26 \%$ varones, $28 \%$ niñas; vestuario $24 \%$ versus $30 \%$; varios $21 \%$ versus $30 \%$ ) (Chi cuadrado $=0,01$, grados de libertad $=4$ ).

2. Contextura de los niños(as) (delgado, normal, obeso e indeterminado) y el sexo de estos. Encontrándose significativamente más niñas delgadas que varones (57\% y 35\% respectivamente) y más varones que niñas en las restantes catego- rías (obesos: $11 \%$ varones versus $10 \%$ niñas; normal 10 versus $6 \%$; indeterminado $43 \%$ versus $26 \%$ ) (Chi cuadrado = 0,003 , grados de libertad $=4$ ).

3. Período de registro (mitad del mes y mitad y fin de semana; fin de mes mitad y mitad y fin de semana y tipo de espacio (comida, vestuario, recreación, y varios) Significativamente en el espacio de comida durante la mitad del mes y en el fin de la semana fue cuando vinieron más niños(as) (43\%); en el espacio de vestuario fue en el fin de mes a fin de semana (33\%); en recreación a mitad del mes durante la mitad de semana (39\%) y en varios, a fin de mes en el fin de semana $(34 \%)$ (Chi cuadrado $=0,000$, grados de libertad $=9)$.

4. Sexo del acompañante y sexo del niño(a). Los acompañantes hombres y solos, vinieron al centro comercial significativamente más con niños que con niñas $(36 \%$ varones y $20 \%$ niñas), lo contrario suce- 
de con las acompañantes mujeres (33\% varones y $55 \%$ niñas) (Chi cuadrado = 0,001 , grados de libertad $=2$ ).

Los varones llegan acompañados por hombres, o mujeres, o por ambos en porcentajes más o menos similares, con un leve predominio de los primeros $(36,2,32,9 \mathrm{y}$ $30,9 \%$, respectivamente); algo muy distinto a lo que acontece con las niñas, puesto que ellas lo hacen de preferencia acompañadas de mujeres $(55,4 \%)$. Cuando los acompañantes son hombre y mujer lo hacen de preferencia junto a un niña y no con una niña $(63,0$ versus $37,0 \%)$.

5. Tipos de espacios y cantidad de compras realizadas. Tomando en cuenta cada uno de los espacios por separado se observa que significativamente en el espacio de comida es la categoría "máximo de consumo" (alta cantidad y/o alta calidad) la que prima (36\%); en el vestuario la categoría "ningún consumo" (58\%); en recreación las categorías "mínimo" y "ninguno" (47 y 50\% respectivamente) y en varios, notablemente, "ningún consumo" $(30 \%)$ (Chi cuadrado $=0,000$, grados de libertad $=6$ )

No obstante lo anterior, el resultado más relevante es que el porcentaje de consumo efectivo es muy bajo, $18,7 \%$ de los casos, el que se incrementa un $23,4 \%$ si se adicionan los casos en los cuales la compra fue mínima; por tanto en un $58 \%$ de los casos no se efectuó consumo alguno.

6. Acto final de compra (inclusión, exclusión) y sexo de los niños(as). Significativamente hubo más varones incluidos en el proceso de compra que niñas (58\% versus $27 \%$ respectivamente) (Chi cuadrado $=0,003$, grados de libertad $=1$ ). En gene- ral hubo significativamente más exclusión que inclusión de niños(as) en el acto final de compras (47\% inclusión versus $53 \%$ exclusión) (Chi cuadrado $=0,003$, grados de libertad $=3$ ).

7. Acto final de compra y el tipo de espacio. Significativamente la mayor inclusión se observó en el espacio de recreación (46\% de niños(as) y la mayor exclusión en el espacio de comida (62\%).

\section{Discusión}

Los centros comerciales están siendo construidos en diversos barrios del gran Santiago, así como en otras ciudades. En este sentido serían una expresión de los alcances que ha adquirido la modernidad, ya que respecto a los mall no reconoce fronteras sociales para su localización. Pero no es menos cierto que aquellos enquistados en los barrios altos y ricos de la ciudad y orientados a esta clase social, aumentan las disparidades socio económico y acrecientan un apartheid social, dado que por su ubicación dificultan el acceso de otras clases sociales. Por tanto se está lejos de la idea, sostenida por ciertos autores, que desean ver a los centros comerciales como un espacio sin clases sociales. Por otro lado, es posible afirmar, a la luz de los resultados de la presente investigación, que los centros comerciales (para los sectores de altos recursos) en su función sociocultural, han alcanzado de alguna manera la importancia de la plaza histórica donde la gente se encuentra para conversar, hacer y mantener contactos, comer y entretenerse, no siendo los niños(as) de las clases pudientes ajenos a este fenómeno. Estos disfrutan del lugar y se encuentran incluidos en el espacio del mall. ${ }^{5}$ Esta emoción placentera, sin mayores contradicciones y con un sentido de plena inclu-

5 Cabe destacar que en la investigación que realizáramos con niños(as) de sectores pobres, observamos que éstos, a diferencia de los niños(as) acomodados, estaban incluidos y también excluidos en el espacio del centro comercial; es decir, en apariencia el niño(a) participa del espacio y las actividades del mall pero no se comporta como un consumidor efectivo en la medida que está impedido por su condición socio económica del consumo real. 
sión, remite al alto valor educativo no formal, que significa para los niños (as), la experiencia -en términos del aprendizaje económico-, el estar en el mall. Más aún, postulamos, que los efectos e influencias de esta experiencia emocional tácita superan los intentos formadores que desea tener la educación formal en los aspectos del aprendizaje económico. Esta aseveración, de alguna manera, entra en confrontación con lo planteado por Abello, Amar y Llanos (1999) cuando postulan que es una educación de calidad aún en las condiciones de precariedad de los niños (as) pobres la que permitiría adquirir habilidades y conocimientos que garanticen posibilidades de inclusión en la vida económica globalizada y con un modelo neoliberal de parte de niños (as) y adultos. Nuestra aseveración no desconoce la importancia de lo educativo, sin embargo enfatiza, que en el ámbito de lo económico, son mas bien los mensajes subliminales, indirectos, no conscientes, los que en última instancia, a nuestro entender, determinan la mayor o menor sofisticación de niños (as) y adultos en los comportamientos económicos.

Cabe destacar (según lo encontrado en la presente investigación) que junto a la observación tan clara de que a los niños (as) de sectores medio alto y alto el espacio del mall les "pertenece", también éstos acatan los requerimientos de los adultos. Es decir, pareciera existir un verdadero contrato social: el niño (a) acata y el adulto fija las normas. El disciplinamiento de los niños (as) se ve reforzado por las características del espacio del mall, lugar ordenado y limpio, donde los guardias controlan. Claros ejemplos de cómo se ha encarnado en los adultos y niños (as) lo normativo, es lo observado en el lugar de alimentación, donde, finalizado el consumo, éstos retiran las bandejas, botan los desperdicios y las ordenan; o cuando los niños (as) no se rebelan al no adquirir lo deseado, acatando los requerimientos de los adultos. ${ }^{6}$ En síntesis, los niños (as) se sienten cómodos en el espacio del centro comercial, y aceptan las normas impuestas por los adultos y el lugar.

Analizando ahora las relaciones estadísticamente significativas entre las variables, cabe destacar aquella en que los varones, más que las niñas, frecuentaban en mayor número los espacios de entretenimiento. En el estudio de Magendzo y Bahamondes (2002) con niños (as) pobres en un centro comercial, ya citado anteriormente, se observó un resultado similar. Se decía entonces, y a modo de hipótesis, que era posible sustentar que culturalmente, los niños (as) han sido socializados en forma diferenciada por sexo respecto a los espacios donde es factible la recreación quedando el ámbito de lo público circunscrito mas bien a lo masculino, no siendo el mall el creador de esta discriminación sino que un lugar que facilita la reproducción de este patrón.

$\mathrm{Al}$ respecto, y en términos generales, se puede sostener que en el mall se reproducen patrones culturales tradicionales. Es decir, es el comportamiento tradicional el que aflora en los centros comerciales. Evidencia de lo anterior son, junto a lo ya enunciada anteriormente, otras tres relaciones encontradas en el presente estudio. Primero, la relación entre sexo y contextura (significativamente más niñas delgadas y varones más obesos), donde la preocupación por la figura queda radicada en las mujeres y no en los hombres. Segundo, la relación entre los niños (as) y el sexo del acompañante (significativamente los hombres concurren al mall acompañando más a varones y las mujeres más a niñas); la práctica social pública se hace, de preferencia, entre personas del mismo sexo. $Y$ tercero, la relación entre el acto de compra efec-

6 Aquí se debe destacar el hecho de que la visita y "participación" en el mall está claramente definida por el momento en que se efectúa la visita vinculado al tema salarios o remuneraciones; no obstante ello refuerza la idea del mall en general como espacio de recreación 
tuado por el menor y el sexo de éste (significativamente más varones incluidos en el acto de compra); nuevamente en el espacio de lo público se muestra mas bien al varón como el responsable del acto de pago.

Visto así los resultados es posible aventurar la hipótesis que la sociedad chilena para los sectores de altos ingresos "ha elegido" el centro comercial como unos de los espacios, junto a otros, para trasmitir valores y comportamientos tradicionales. $\mathrm{O}$, dicho de otra manera, se da una interdependencia casi perfecta entre lo que el mall transmite y perpetua y aquello que los adultos conscientes o inconscientemente desean comunicar a los niños(as) en el dominio económico; es decir, conforman con el espacio, por tanto se adecuan al espacio sin generar formas alternativas de estar él.

Desde los resultados obtenidos, es posible especular a modo de una hipótesis tentativa que estos niños(as) serán los naturales adultos que ocuparán los espacios de los centros comerciales sin mayores contradicciones.

Por otro lado, en el desarrollo social y cognitivo de los niños(as) es posible afirmar, en términos generales, que operan dos procesos: por una parte, los estímulos (sensaciones e información) que provienen del entorno o contexto en el que se desenvuelven éstos $\mathrm{y}$, por otra parte, la etapa o fase de desarrollo cognitivo por la que atraviesan. Esta última se supone que es decisiva en el proceso de organizar y elaborar los elementos que el niño(a) reconoce en el entorno.

La problemática abordada en el presente estudio trató justamente de identificar de manera más precisa las formas cómo operan estos dos procesos en un ámbito específico de la formación de los niños(as) que es lo económico, tanto en lo general, como lo particular en la condición de consumidor.

Los resultados permiten sostener que las conductas desarrolladas por el niño(a) en el mall están inscritas en un proceso general de formación que desde las orientaciones de parte de los adultos sigue un patrón gradual de incorporación de los niños(as) hacia la condición plena de consumidor. Es decir, un individuo capaz de integrar información respecto a lo económico, sobre el concepto del dinero o medios para adquirir bienes o servicios (elección sobre aquello que quiere adquirir), información de la oferta de bienes (tipos de bienes y servicios y sus respectivos precios), e información del aporte "adicional" que desde el punto de vista social (estatus o prestigio) puede brindarle la adquisición de los bienes.

En otras palabras, la forma que asume el acto de consumo en los niños(as) se caracteriza, primero, por tener grados diferenciados de participación en los ritos que éste conlleva: acercamiento al espacio; reconocimiento de objetos (constatación de sus virtudes; comparación entre objetos), decisión de qué consumir; cierre de la transacción económica (dependiendo del tipo de espacio donde se consume), segundo, lo anterior se caracteriza por ser un proceso gradual en que se permiten quiebres (en este espacio se participa, en este otro no se participa) en la medida que está asociado a categorías de acción social que definen un comportamiento distinto (por ejemplo, en el espacio de juego es factible participar en el proceso de consumo completo; y en otros -espacio de comida, vestuario, donde se restringe su involucramiento, donde las condiciones para su realización como acto "real", pleno, aun están ausentes o veladas. Con ello el niño(a) reconoce la posición que ocupa como consumidor en ese momento $y$ cuando es factible una $u$ otra conducta. Por lo tanto, se evidencia que los adultos marcan las etapas del desarrollo del niño(a) como consumidor.

Si lo anterior es cierto quiere decir que los esquemas que elaboran los niños(as) respecto al mundo no queda circunscrito a la dimensión cognitiva en interacción con las señales del entorno, sino que están reguladas por los esquemas que los adultos poseen so- 
bre como debe ser el comportamiento del niño(a) en determinadas etapas de su desarrollo económico. En otras palabras estamos en presencia de la construcción de esquemas dentro de esquemas. De esa manera las senales del entorno, en estricto rigor no pueden ser asumidas como no elaboradas, si no que en su totalidad buena parte de ellas se presentan al niño(a) como imágenes ya estructuradas a partir de los esquemas manejados por los adultos y los recintos donde se desplazan.

Es esto lo que permite plantearse la posibilidad de que el niño(a) puede "transitar" en el mall, de un espacio a otro desempeñando en cada uno de ellos involucramientos distintos, sin manifestar mayor contrariedad, puesto que las señales del entorno ya vienen elaboradas; no es que éste se le presente como un conjunto amplio de "datos" que puede ordenar considerando la etapa cognitiva por la que atraviesa; los datos ya están ordenados, dejando poco espacio para la acción autónoma del niño(a). Si esto es así, se puede concluir que, por una parte, el concurrir en forma reiterada a un centro comercial no hace otra cosa que establecer esa forma como la única de comportarse en la adquisición de bienes y servicios y, por otra parte, la posibilidad de abrir el comportamiento del niño(a) a otra experiencia de lo económico sólo sería factible en el caso que los adultos enfrenten un escenario en el cual carezcan de mecanismos efectivos para construir y resolver lo económico.

La comprensión de lo anterior se enmarca en lo elaborado por Maturana (1990) cuando dice que lo humano se constituye en el entrelazamiento de lo emocional con lo racional. Surge entonces el concepto de "esquema emocional" o función de procesos automáticos que generan las respuestas tonales de los individuos. Es decir, los esquemas emocionales son los que incorporan experiencias innatas o aprendidas y proveen información crucial que no es la prepositiva (sensorial-perceptiva o cognitiva) para guiar la experiencia de la vida en conciencia. Este flujo de concienciación incorpora ambos cognición y afectó, pero no siempre implica un proceso conceptual reflexivo (Greenberg, Speltz, Deklyen 1993). Los esquemas emocionales entonces son una rica combinación de nuestra biología, nuestra experiencia y nuestra cultura, proveyendo altos niveles de integración en nuestra razón -vital del experienciar (Greemberg; 1991). Lo dicho tiene directa relación con nuestros hallazgos, en el sentido que postulamos que la experiencia de los niños(as) de medio alto y alto en el centro comercial tiene un impacto psicosocial profundo y tácito en ellos lo que va a determinar su comportamiento social y económico en la vida adulta.

\section{Referencias}

Abello, R., Amar, J. \& Llanos, M. (1999). Desarrollo de conceptos económicos en niños y adolescentes colombianos y su interacción en los sectores educativos y Calidad de vida. Proyecto CIDHUM. Universidad del Norte, Conciencias y D. I. P.: Barranquilla.

Amar, J. (2002). Las representaciones sociales de los conceptos económicos en niños que viven en contextos de pobreza. Centro de Investigaciones en Desarrollo Humano CIDHUM División de Humanidades y Ciencias Sociales Universidad del Norte.: Barranquilla.

Bourdieu, P. (2002) Las Estructuras Sociales de la Economía. Manantial Buenos Aires Primera reimpresión

Certaux M. D.(1996). La Invención de lo cotidiano. A. C. México: Universidad Iberoamericana

Delval, J.( 1989)." La Representación Infantil del Mundo Social". El Mundo Social en la mente Infantil. Compilación de Elliot T., Ileana E. y Josetxu, L. Alianza Editorial: Madrid. 
Denegri, M., Keller, A., Ripoli, M., Palavecinos, M. \& Delval, J. (1997). La construcción de representaciones sociales acerca de la pobreza y desigualdad social en la infancia y la adolescencia. Universidad de la Frontera: Chile.

Denegri, M. (1998) La construcción de conceptos económicos. ¿Cómo comprenden los niños y adolescentes la economía? Boletín de la Red para la educación del consumidor, $N 3$ julio: Chile.

Greenberg, M.T., Speltz, M.L., \& Deklyen, M. (1993) The role of attachment in the early development of disruptive problems. Development and Psy chopathology, (5) 191-213.

Greenberg , L.S. (1991) Research on the processof change, Psychotherapy reserch,1,14-24
Magendzo, S. y Bahamondes, M. (2002) La Participación de los Niños Pobres en el "Espectáculo" del Consumo. Castalia, Revista de Psicología Universidad Academia de Humanismo Cristiano. $N$ 3. Vol. 1 pág. 97 112 Santiago Chile.

Maturana, H. (1990). Emoción y Lenguaje en Educación y Politica: Santiago: Colección Hachette/ Comunicación.

Moulián, T. (1998) El Consumo me Consume. Santiago: LOM Ediciones.

Sarlo, B. (1996) Escenas de la vida Posmoderna. Intelectuales, Arte y Video Cultura en la Argentina. Buenos Aires: Ed. Arie.

Webley, P. Lea, S.E.G. (1993) The Partial Unacceptability of Money as Repayment of Neighborly Help.: Human Relation 46. 